This is the final peer-reviewed accepted manuscript of:

Effect of sterilization and crosslinking on gelatin films. In JOURNAL OF MATERIALS SCIENCE. MATERIALS IN MEDICINE pp.1-9 2015.- ISSN:0957-4530 vol. 26 (2) Amadori, S.; Torricelli, P.; Rubini, K.; Fini, M.; Panzavolta, S.; Bigi, A.

The final published version is available online at: DOI:10.1007/s10856-015-5396-4

Rights / License:

The terms and conditions for the reuse of this version of the manuscript are specified in the publishing policy. For all terms of use and more information see the publisher's website.

This item was downloaded from IRIS Università di Bologna (https://cris.unibo.it/)

When citing, please refer to the published version. 


\title{
Effect of sterilization and crosslinking on gelatin films
}

\author{
Sofia Amadori • Paola Torricelli • Katia Rubini • \\ Milena Fini $\cdot$ Silvia Panzavolta $\cdot$ Adriana Bigi
}

\begin{abstract}
Sterilization through $\gamma$-irradiation has been reported to affect collagen mechanical properties, but its possible effects on gelatin based materials have not been investigated up to now. Herein we report the results of a mechanical, chemical and thermal study performed on gelatin films before and after $\gamma$-irradiation. The investigation was performed on uncrosslinked films as well as on crosslinked films. To this aim, two common crosslinking agents, glutaraldehyde and genipin, at different concentration $(0.15,0.30$ and $0.67 \%)$ were used. The results indicate that sterilization significantly affects the mechanical properties of uncrosslinked films, whereas it displays a modest effect on gelatin swelling, release in solution, thermal stability and molecular structure. Both glutaraldehyde and genipin enhance the mechanical properties and stability in solution of the gelatin films. In particular, the values of Young modulus increase as a function of
\end{abstract}

S. Amadori · K. Rubini $(\bowtie) \cdot$ S. Panzavolta $(\bowtie) \cdot$ A. Bigi Department of Chemistry "G. Ciamician”, University of Bologna, Bologna, Italy

e-mail: katia.rubini@unibo.it

S. Panzavolta

e-mail: silvia.panzavolta@unibo.it

P. Torricelli · M. Fini

Laboratory of Biocompatibility, Technological Innovations and Advanced Therapies, Department Rizzoli RIT (Research, Innovation and Technology), Rizzoli Orthopaedic Institute-IOR, Bologna, Italy

P. Torricelli · M. Fini

Laboratory of Preclinical and Surgical Studies, Codivilla-Putti Research Institute, Rizzoli Orthopaedic Institute, Bologna, Italy

A. Bigi

National Consortium of Materials Science and Technology

(INSTM, Bologna RU), University of Bologna, Bologna, Italy crosslinker concentration up to about 10 and $18 \mathrm{MPa}$ for genipin and glutaraldehyde treated samples respectively. The results of in vitro study demonstrate that the films crosslinked with genipin do not display any cytotoxic reaction, whereas glutaraldehyde crosslinking provokes an acute and dose dependent cytotoxic effect.

\section{Introduction}

Gelatin is a biodegradable, highly compatible, abundant and cheap raw material, which can be successfully applied in tissue engineering, drug delivery, wound dressing and gene therapy [1, 2]. In fact gelatin numerous applications include packaging, food, pharmaceutical and photographic industry, as well as production of materials addressed to the biomedical field. It is obtained from collagen, through processes implying breaking of the protein triple helix molecules into random coils. Cooling gelatin aqueous solutions results in a partial renaturation of the collagen molecular structure, which involves a conformational disorder-order transition of the chains [3-5]. The partial recovery of the triple helix structure during the sol-gel transformation has been extensively investigated with different techniques, including differential scanning calorimetry, circular dichroism, X-ray diffraction and infrared spectroscopy [6-10]. At variance with collagen, gelatin does not express antigenity. Nonetheless, for safety reasons also gelatin based biomaterials are submitted to sterilization. Gamma irradiation has been introduced as the simplest and most effective procedure of sterilization without toxic residues [11]. However, $\gamma$-irradiation has been reported to cause remarkable modifications of collagen-based materials. Collagen chain scission, decrease of tensile strength and modulus, reduced thermal stability, are among the reported undesired effects [11-13]. These 
adverse side effects are due to the high relative irradiation doses used for collagenous materials [12] and allografts [13]. Even if the doses generally utilized for gelatin based materials are not particularly high [14-16], possible modifications induced by sterilization cannot be excluded a priori because of the significant lower stability of gelatin with respect to collagen. However, no detailed information on the possible alterations induced by $\gamma$-irradiation on gelatin are known. In this study we investigated the effect of sterilization performed by $\gamma$-irradiation on the mechanical, structural and stability properties of gelatin films. Since gelatin is highly soluble in aqueous solution, its materials for biomedical applications must be submitted to crosslinking. To this aim, we used two different crosslinking agents, glutaraldehyde (GTA) and genipin (GEN), at increasing concentrations, and analyzed the effects of sterilization on uncrosslinked, as well as on crosslinked films. Moreover, the cytotoxicity of the crosslinked films was evaluated using BJ5ta human fibroblasts.

\section{Materials and methods}

Type A gelatin (280 Bloom Italgelatine S.p.A.) from pig skin was used. Gelatin films were prepared from a $5 \%$ aqueous gelatin solution. Films were obtained on the bottom of Petri dishes (diameter $=6 \mathrm{~cm}$ ) after water evaporation at room temperature from $10 \mathrm{ml}$ of gelatin solution [17]. After air drying, films were crosslinked either with $10 \mathrm{ml}$ of genipin (Wako, Japan) solutions or with $10 \mathrm{ml}$ of GTA solutions at different concentrations [18, 19]. The concentrations of the crosslinking agents were $0.15,0.30$ and $0.67\left(\mathrm{wt} \% \mathrm{~V}^{-1}\right)$. Both the crosslinking solutions were prepared in phosphate buffer $0.1 \mathrm{M} \mathrm{pH} 7.4$. After $24 \mathrm{~h}$ at room temperature, the crosslinked samples were washed in $0.1 \mathrm{M}$ glycine aqueous solution for $30 \mathrm{~min}$, and then repeatedly rinsed with double distilled water and air dried at room temperature.

Crosslinked samples were labeled GEN0.15, GEN0.30, GEN0.67, GTA0.15, GTA0.30 and GTA0.67. Control samples of uncrosslinked gelatin were labeled G5.

Some gelatin films were sterilized with gamma rays (cobalt-60) at a dose of $25 \mathrm{kGy}$.

\subsection{Mechanical tests}

Stress-strain curves were recorded using an INSTRON Testing Machine 4465 and the Series IX software package. Strip-shaped $(3 \mathrm{~mm} \times 30 \mathrm{~mm})$ air-dried films were immersed in a mixture of water and ethanol in a 2:3 ratio (constant relative humidity of $75 \%$ ) for $72 \mathrm{~h}$ and stretched in a mixture of the same composition using a crosshead speed of $5 \mathrm{~mm} \mathrm{~min}{ }^{-1}$ [17]. The thickness of the wet samples was determined using a Leitz SM-LUX-POL microscope. Young's modulus, $E$, the stress at break, $\sigma_{\mathrm{b}}$, and the strain at break, $\varepsilon_{\mathrm{b}}$, of the crosslinked strips were measured.

Statistical analysis was performed on the data reported in Table 1. Each value is the mean of seven determinations and is reported with its standard deviation. Single factor analysis of variance (ANOVA) was employed to assess statistical significance of the results. A value of $P<0.005$ was considered statistically significant.

\subsection{Differential scanning calorimetry}

Calorimetric measurements were performed using a Perkin Elmer Pyris Diamond DSC equipped with a model ULSP intracooler. Temperature and enthalpy calibration were performed using high-purity standards ( $n$-decane and indium). The film samples were examined under air-dried conditions. The measurements were carried out on known amounts of gelatin films (3-4 mg) and the samples were hermetically sealed in aluminum pans. Heating was carried out at $5{ }^{\circ} \mathrm{C} \mathrm{min}{ }^{-1}$ from 40 to $110{ }^{\circ} \mathrm{C}$. Denaturation temperature $\left(T_{\mathrm{D}}\right)$ was determined as the peak value of the corresponding endothermic event. The value of denaturation enthalpy $\left(\Delta H_{\mathrm{D}}\right)$ was calculated with respect to the weight of air-dried gelatin.

\subsection{Determination of extent of crosslinking}

The extent of crosslinking of gelatin films was determined by a UV assay of the uncrosslinked $\varepsilon$-amino groups before and after reaction with genipin or glutaraldehyde [20]. Following reaction with $0.5 \%$ trinitrobenzensulfonic acid (TNBS), gelatin was hydrolysed with $6 \mathrm{M} \mathrm{HCl}$, and extracted with ethyl ether. The absorbance of the diluted solution was measured at $346 \mathrm{~nm}$ in a Cary 50 Bio spectrophotometer against a blank. The relationship between absorbance and moles of e-amino groups per gram of gelatin is:

moles of e-amino groups/g gelatin

$$
=\frac{2(\text { absorbance })(0.0201)}{\left(1.46 \times 10^{4} 1 / \mathrm{mol} \mathrm{cm}\right)(b)(x)}
$$

where $1.46 \times 10^{4} 1 \mathrm{~mol}^{-1} \mathrm{~cm}^{-1}$ is the molar absorptivity of TNP-lys, $b$ is the cell path length in centimeter, and $x$ is the sample weight in grams.

\subsection{Release of gelatin}

$50 \mathrm{mg}$ of gelatin film was immersed in $5 \mathrm{ml}$ of PBS $0.1 \mathrm{M}$, $\mathrm{pH} 7.4$, at $37{ }^{\circ} \mathrm{C}$. Fractions were collected and analyzed for gelatin content after $6 \mathrm{~h}, 1,2,7,14,21,28$ days. Gelatin concentration in the release buffer was determined by colorimetric assay using a bicinchoninic acid protein assay kit 
[21] (Sigma Chemical Co., St. Louis, MO, USA). A $4 \%$ copper(II) sulphate pentahydrate solution was mixed with an excess of bicinchoninic acid at a final ratio of 1:50 v/v; $300 \mu \mathrm{l}$ of the release solution was added to $2 \mathrm{ml}$ of the assay solution in a test tube. Following further addition of PBS up to a final volume of $5 \mathrm{ml}$, the solutions were cooled to room temperature and the absorbance of each solution at $562 \mathrm{~nm}$ was measured using a Cary 50 Bio spectrophotometer. The gelatin concentration in the release solution was determined through comparison with a calibration curve.

\subsection{Swelling}

Gelatin films were weighed in air-dried conditions. Then, they were immersed in PBS 0.1 M at pH 7.4 for different periods of time. Wet samples were wiped with filter paper to remove excess liquid and reweighed. The amount of adsorbed water was calculated as

$W \%=\frac{\left(W_{w}-W_{d}\right) \times 100}{W_{d}}$

where $W_{w}$ and $W_{d}$ are respectively the weights of the wet and the air-dried samples.

\subsection{Attenuated total reflection Fourier transform infrared analysis (ATR-FTIR)}

FT-IR spectra were collected with a Thermo Nicolet 380 spectrometer equipped with ATR accessory and the spectra resolution was $4 \mathrm{~cm}^{-1}$. The spectrum of the sample was obtained by placing a film onto germanium crystal without any additional sample preparation. The spectra were the result of 196 scans.

\subsection{In vitro studies}

\subsubsection{Cell culture}

The BJ-5ta human fibroblast cell line (ATCC, CRL-4001) was cultured according to ATCC recommendations [four parts high glucose Dulbecco's Modified Eagle's Medium (DMEM) containing $1.5 \mathrm{~g} \mathrm{l}^{-1}$ sodium bicarbonate and one part of Medium 199, supplemented with $10 \%(\mathrm{v} / \mathrm{v})$ foetal bovine serum (FBS), and $0.01 \mu \mathrm{m} \mathrm{m}^{-1}$ hygromycin B]. The fibroblasts were maintained at $37^{\circ} \mathrm{C}$ in a humidified atmosphere of $5 \% \mathrm{CO}_{2}$ and medium was replaced every 2 days. At $90 \%$ confluence, cells were detached from culture flasks by trypsinization, and cell number and viability were checked by trypan blue dye exclusion test. Fibroblasts were plated at a density of $5 \times 10^{4}$ cells ml ${ }^{-1}$ in 12-well plates containing sterile samples for each of gelatin film treated with different crosslinking (GTA0.15, GTA0.30, GTA0.67, GEN0.15, GEN0.30, GEN0.67) and in wells for negative (CTR-, medium only) and positive $(\mathrm{CTR}+$, medium $+0.05 \%$ phenol solution) controls. Plates were cultured in standard conditions, at $37 \pm 0.5{ }^{\circ} \mathrm{C}$ with $95 \%$ humidity and $5 \% \pm 0.2 \mathrm{CO}_{2}$ up to 28 days.

\subsubsection{Cytotoxicity evaluation}

The quantitative evaluation of cytotoxicity was performed by measuring cell proliferation, lactate dehydrogenase enzyme (LDH) release, and pro-inflammatory cytokine and growth factor production.

Cell proliferation and viability at $72 \mathrm{~h}, 14$ and 28 days was assessed by WST1 (WST1, Roche Diagnostics GmbH, Manheim, Germany) colorimetric reagent test. The assay is based on the reduction of tetrazolium salt a soluble formazan salt by a reductase of the mitochondrial respiratory chain, active only in viable cells. $100 \mu \mathrm{l}$ of WST1 solution and $900 \mu \mathrm{l}$ of medium (final dilution: 1:10) were added to the cell monolayer, and the multi-well plates were incubated at $37{ }^{\circ} \mathrm{C}$ for a further $4 \mathrm{~h}$. Supernatants were quantified spectrophotometrically at $450 \mathrm{~nm}$ with a reference wavelength of $625 \mathrm{~nm}$. Results of WST1 are reported as optical density (OD) and directly correlate with the cell number. Proliferation percent relative to CTR - at $24 \mathrm{~h}$ are also reported. At the end of experimental times the supernatant was collected from all wells and centrifuged to remove particulates, if any, for LDH (LDH enzyme-kinetic test, Roche, D), Interleukin1 $\beta$ (IL1 $\beta$, e-Bioscience Bender Medsystems, Vienna, A), and tumor necrosis factor $\alpha$ (TNF $\alpha$, Boster, Fremont, CA, USA) release.

\subsubsection{Cell morphology}

Samples for each material, at the end of the experiment, were processed for scanning electron microscopy (SEM): osteoblasts grown on the materials were fixed in $2.5 \%$ glutaraldehyde, in $\mathrm{pH} 7.4$ phosphate buffer $0.01 \mathrm{M}$ for $1 \mathrm{~h}$ and dehydrated in a graded ethanol series. After a passage in hexamethyldisilazane, the samples were air dried. The samples were sputter-coated with Pd prior to examination with a Philips CM100 scanning electron microscope.

\subsubsection{Statistical analysis}

Statistical evaluation of data was performed using the software package SPSS/PC+StatisticsTM 21 (SPSS Inc., Chicago, IL, USA). The study is the results of three independent experiments and data are reported as mean \pm standard deviations (SD) at a significance level of $P<0.05$. After having verified normal distribution and homogeneity of variance, a one-way ANOVA was done for comparison between groups. Finally, post hoc multiple 
comparison test (Dunnett) was performed to detect significant differences among groups and controls.

\section{Results and discussion}

Materials for biomedical applications need sterilization before use. Sterilization through $\gamma$-irradiation has been reported to cause a severe decrease of the mechanical strength collagen fibers, presumably by inducing chain scissions via radical formation [12]. In agreement, the results of this study indicate that the mechanical properties of uncrosslinked gelatin films are affected by sterilization. The stress-strain curve of uncrosslinked gelatin films is characterized by an initial elastic region at relatively high modulus, followed by a yield region and then by a region at slightly increased modulus (Fig. 1). Sterilization provokes a significant $(P<0.005)$ reduction of stress at break, $\sigma_{\mathrm{b}}$, and strain at break, $\varepsilon_{\mathrm{b}}$, without major modifications of the shape of the curve (Fig. 1). On the other hand, crosslinking causes an abrupt change in the shape of the curve (data not shown), as previously reported $[18,19]$. Both genipin and glutaraldehyde crosslinked films display a significant

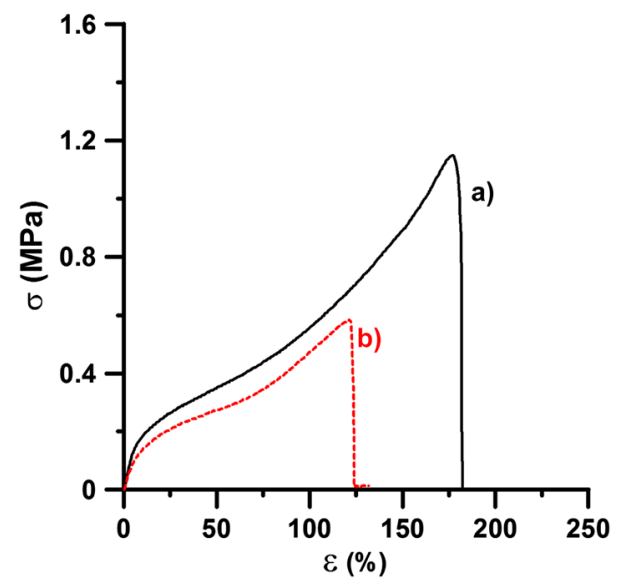

Fig. 1 Typical stress-strain curves recorded from uncrosslinked gelatin films (G5): (a) before and (b) after sterilization increase of Young modulus and decrease of breaking strain $(P<0.005)$ with respect to uncrosslinked gelatin films (Table 1). In particular, the values of Young modulus increase as a function of crosslinker concentration up to about 10 and $18 \mathrm{MPa}$ for genipin and glutaraldehyde treated samples respectively. The effect of crosslinking on breaking stress is less relevant, as previously reported [18, 19]. Sterilized crosslinked samples generally display lower values of the mechanical parameters than non-sterilized samples. However, the differences are not significant $(P>0.2)$, suggesting that the improvement of the mechanical performance due to crosslinking plays a major role with respect to the possible negative effects of sterilization. The mechanical properties of the different samples are in agreement with their different extent of crosslinking, which has been calculated from the moles of free-amino groups per gram of gelatin [20]. The results reported in Table 2 indicate that the extent of crosslinking increases as a function of the chemical agent concentration and displays slightly higher values for GTA than for GEN treated samples. Sterilization provokes a modest decrease of the crosslinking degree. Crosslinking greatly influences gelatin swelling. In fact, gelatin undergoes a considerable swelling in PBS solution, up to about 700 and $1,300 \%$ after 1 and

Table 2 Extent of crosslinking, expressed as percent of free $\varepsilon$-amino groups lost after crosslinking as a function of crosslinker concentration

\begin{tabular}{lll}
\hline Sample & \multicolumn{2}{l}{ Extent of crosslinking $(\%)$} \\
\cline { 2 - 3 } & Before sterilization & After sterilization \\
\hline GEN0.15 & $34 \pm 2$ & $29 \pm 4$ \\
GEN0.30 & $60 \pm 4$ & $58 \pm 4$ \\
GEN0.67 & $89 \pm 3$ & $85 \pm 3$ \\
GTA0.15 & $42 \pm 1$ & $40 \pm 4$ \\
GTA0.30 & $75 \pm 1$ & $72 \pm 3$ \\
GTA0.67 & $94 \pm 1$ & $90 \pm 3$ \\
\hline
\end{tabular}

Each value is the mean of three determinations and is reported with its standard deviation
Table 1 Strain at break, $\varepsilon_{\mathrm{b}}$, stress at break, $\sigma_{\mathrm{b}}$, and Young's modulus, $E$, of non-sterilized and sterilized gelatin films, examined at RH $75 \%$

Each value is the mean of seven determinations and is reported with its standard deviation

\begin{tabular}{lccccccc}
\hline Sample & \multicolumn{2}{l}{ Before sterilization } & & \multicolumn{4}{l}{ After sterilization } \\
\cline { 2 - 3 } & $E(\mathrm{MPa})$ & $\sigma(\mathrm{MPa})$ & $\varepsilon(\%)$ & & $E(\mathrm{MPa})$ & $\sigma(\mathrm{MPa})$ & $\varepsilon(\%)$ \\
\hline GEL & $2.9 \pm 0.4$ & $1.1 \pm 0.3$ & $192 \pm 27$ & & $2.5 \pm 0.3$ & $0.5 \pm 0.2$ & $127 \pm 20$ \\
GEN0.15 & $4.8 \pm 0.3$ & $1.7 \pm 0.6$ & $30 \pm 7$ & & $4.4 \pm 0.3$ & $1.5 \pm 0.2$ & $31 \pm 5$ \\
GEN0.30 & $7.1 \pm 0.4$ & $1.8 \pm 0.4$ & $22 \pm 4$ & & $6.8 \pm 0.3$ & $1.5 \pm 0.2$ & $20 \pm 5$ \\
GEN0.67 & $10.3 \pm 0.7$ & $1.6 \pm 0.4$ & $16 \pm 4$ & & $9.7 \pm 0.4$ & $1.5 \pm 0.2$ & $13 \pm 4$ \\
GTA0.15 & $6 \pm 1$ & $1.8 \pm 0.3$ & $29 \pm 7$ & & $5.6 \pm 0.6$ & $1.5 \pm 0.4$ & $27 \pm 7$ \\
GTA0.30 & $16 \pm 1$ & $2.2 \pm 0.3$ & $14 \pm 4$ & & $14 \pm 2$ & $2.0 \pm 0.4$ & $15 \pm 7$ \\
GTA0.67 & $18 \pm 3$ & $2.9 \pm 0.6$ & $14 \pm 2$ & & $17 \pm 1$ & $2.5 \pm 0.2$ & $13 \pm 2$ \\
\hline
\end{tabular}



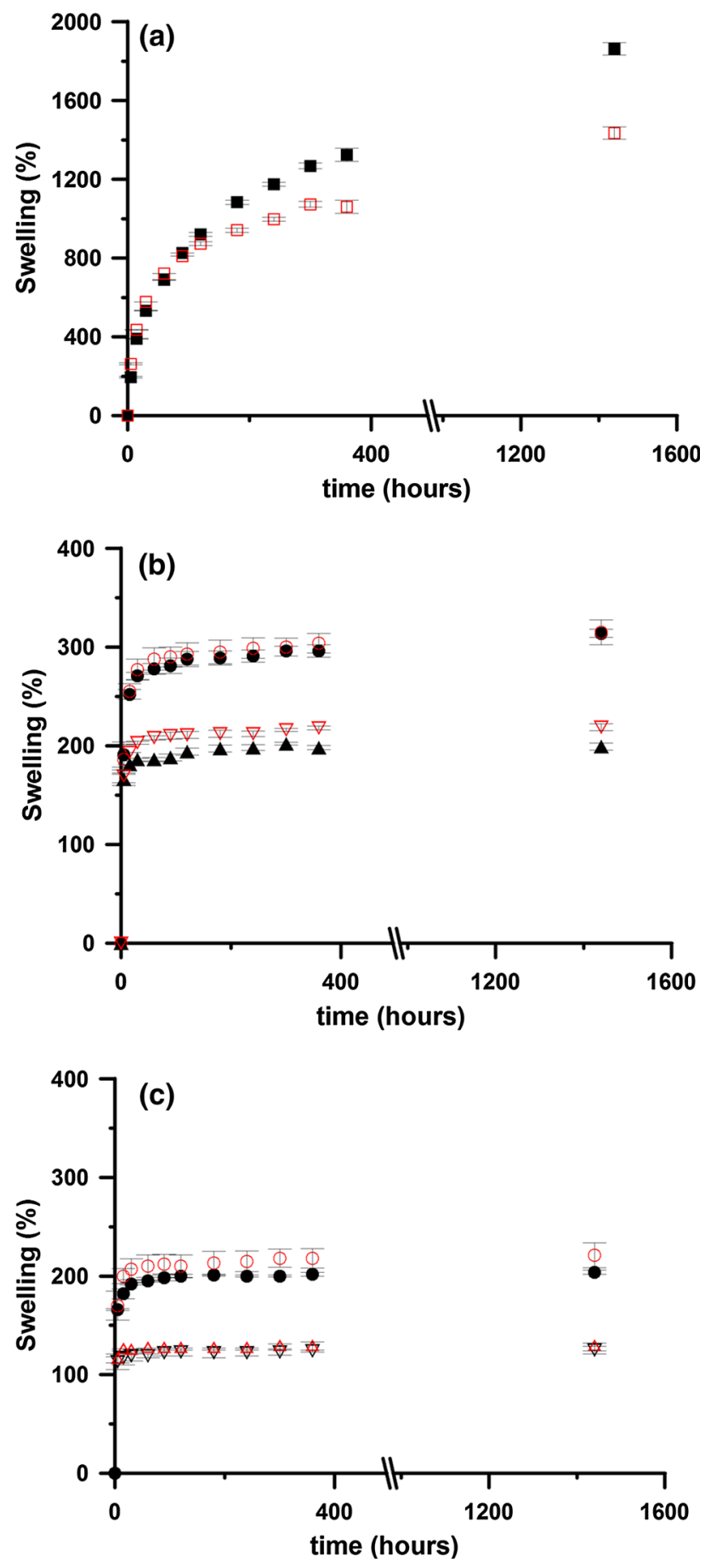

Fig. 2 Swelling curves of (a) G5: (filled square) before and (empty square) after sterilization; (b) GEN0.15: (filled circle) before and (empty circle) after sterilization, GEN0.67 (filled triangle) before and (empty inverted triangle) after sterilization; (c) GTA0.15: (filled circle) before and (empty circle) after sterilization, GTA0.67 (empty inverted triangle) before and (empty triangle) after sterilization. Each value was determined in triplicate and reported with its standard deviation

$6 \mathrm{~h}$ respectively (Fig. 2). Even low crosslinker concentrations drastically reduce swelling. In particular, the degree of swelling decreases on increasing the chemical agent

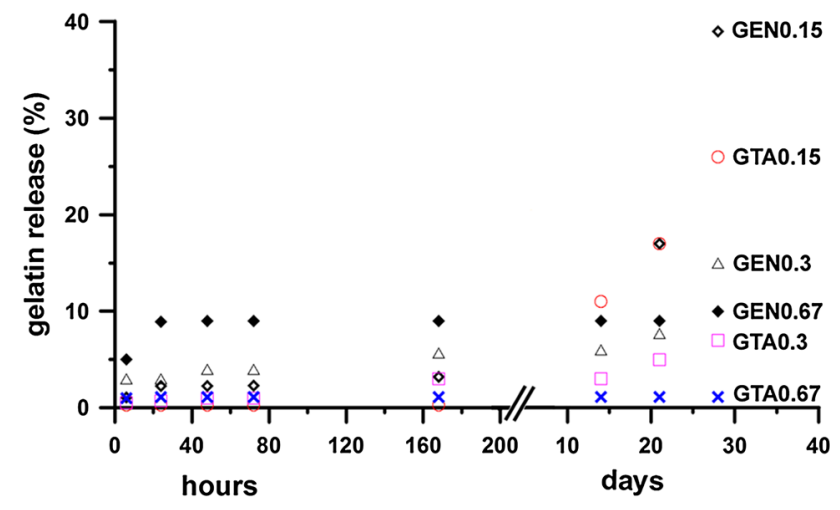

Fig. 3 Gelatin release from genipin and glutaraldehyde treated samples before sterilization. Each value was determined in triplicate and reported with its standard deviation

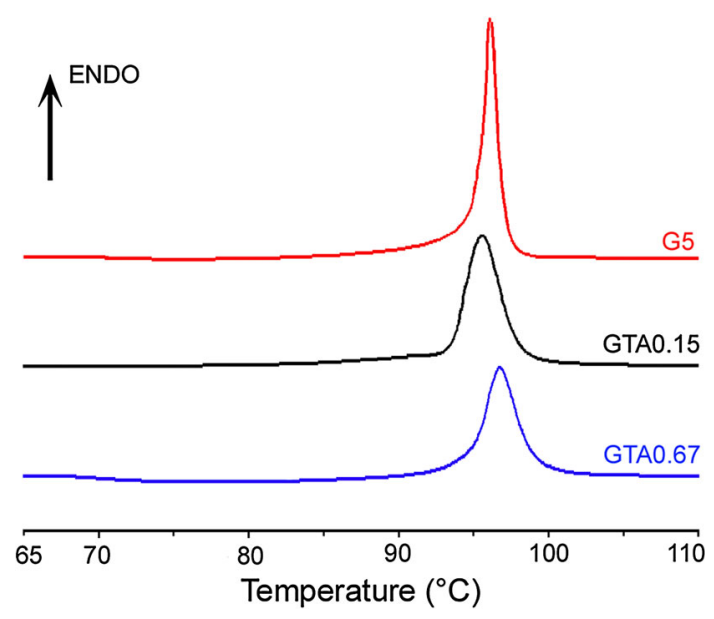

Fig. 4 DSC plots of G5, GTA0.15 and GTA0.67 films

Table 3 Denaturation temperature, $T_{\mathrm{D}}$ and denaturation enthalpy, $\Delta H_{\mathrm{D}}$, of crosslinked gelatin films as a function of crosslinker concentration

\begin{tabular}{llllll}
\hline Sample & \multicolumn{2}{l}{ Before sterilization } & & \multicolumn{2}{l}{ After sterilization } \\
\cline { 2 - 3 } \cline { 5 - 6 } & $T_{\mathrm{D}}\left({ }^{\circ} \mathrm{C}\right)$ & $\Delta H_{\mathrm{D}}\left(\mathrm{J} \mathrm{g}^{-1}\right)$ & & $T_{\mathrm{D}}\left({ }^{\circ} \mathrm{C}\right)$ & $\Delta H_{\mathrm{D}}\left(\mathrm{J} \mathrm{g}^{-1}\right)$ \\
\hline GEL & $96 \pm 1$ & $29 \pm 1$ & & $94 \pm 1$ & $28 \pm 1$ \\
GEN0.15 & $98 \pm 1$ & $17 \pm 1$ & & $98 \pm 1$ & $17 \pm 1$ \\
GEN0.30 & $96 \pm 1$ & $14 \pm 1$ & $96 \pm 1$ & $14 \pm 1$ \\
GEN0.67 & $96 \pm 1$ & $12 \pm 1$ & $96 \pm 1$ & $12 \pm 1$ \\
GTA0.15 & $96 \pm 1$ & $20 \pm 1$ & $96 \pm 1$ & $19 \pm 1$ \\
GTA0.30 & $96 \pm 1$ & $16 \pm 1$ & $96 \pm 1$ & $16 \pm 1$ \\
GTA0.67 & $97 \pm 1$ & $15 \pm 1$ & $96 \pm 1$ & $16 \pm 1$ \\
\hline
\end{tabular}

Each value is the mean of three determinations and is reported with its standard deviation

concentration down to values around $200 \%$ for genipin crosslinked films and to values around $100 \%$ for glutaraldehyde crosslinked films, after $6 \mathrm{~h}$ immersion. The same 
values are maintained after 1 day in PBS solution, when the degree of swelling of uncrosslinked films reaches a mean value higher than $1,800 \%$. Sterilization reduces swelling of uncrosslinked gelatin, whereas it provokes modest variations in the swelling behavior of crosslinked samples, as shown in Fig. 2. In agreement, gelatin release in PBS is not affected by sterilization. Gelatin release is reported in Fig. 3. At low crosslinker concentrations, the release of gelatin is very low up to 1 week, after which it increases as a function of time up to about 40 and $25 \%$ for

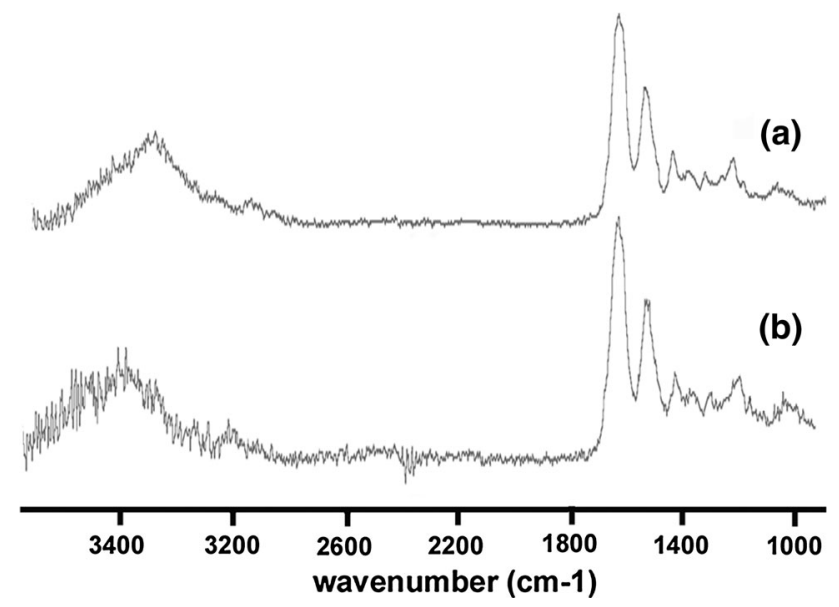

Fig. 5 FT-IR spectra of gelatin films (G5) before and after sterilization. (a) Before sterilization; (b) after sterilization
GEN0.15 and GTA0.15 respectively. A similar trend can be observed for GEN0.30 and GTA0.30, although in this case at 28 days the release amounts to just 15 and $7 \%$ respectively. At variance, gelatin release at the highest crosslinker concentration is very small, and it does not vary with time. The initial relative high values of gelatin release from GEN0.67 could be an artifact caused by genipin release, as indicated by the blue coloration assumed by the PBS solution soon after GEN0.67 immersion.

The DSC plot of gelatin (Fig. 4) shows an endothermic peak due to the helix-coil transition of collagen. The value of the denaturation enthalpy associated to this peak is related to the relative amount of triple helical structure, and it depends on the extent of hydrogen bonds, which break endothermically, and of covalent crosslinks, which break exothermically [7]. In agreement with previous data [18, $19,22]$ crosslinking provokes a decrease of the denaturation enthalpy (Table 3), due both to a reduction of hydrogen bonds, and to a simultaneous increase in the extent of covalent crosslinks. The DSC plots of G5, GTA0.15 and GTA0.67 reported in Fig. 4 allow to appreciate the decrease of the relative area of the endothermic peak on increasing crosslinking concentration. At variance with the values of denaturation enthalpy, which decrease as the concentration of the crosslinking agents increases, the values of denaturation temperature are not affected by crosslinking. Sterilization does not seem to affect the
Fig. 6 Fibroblast BJ5ta proliferation after (a) $72 \mathrm{~h}$, (b) 14 (first column) and 28 (second column) days of culture on material samples and controls. Results are the mean \pm SD (post hoc Dunnett test: $* P<0.05 ; * * P<0.005)$. (a) *GTA0.67 versus CTR-; $* * \mathrm{CTR}+$ versus all samples. (b) 14 days: **GTA0.67 versus CTR-;28 days: *GTA0.67, GEN0.67 versus CTR-
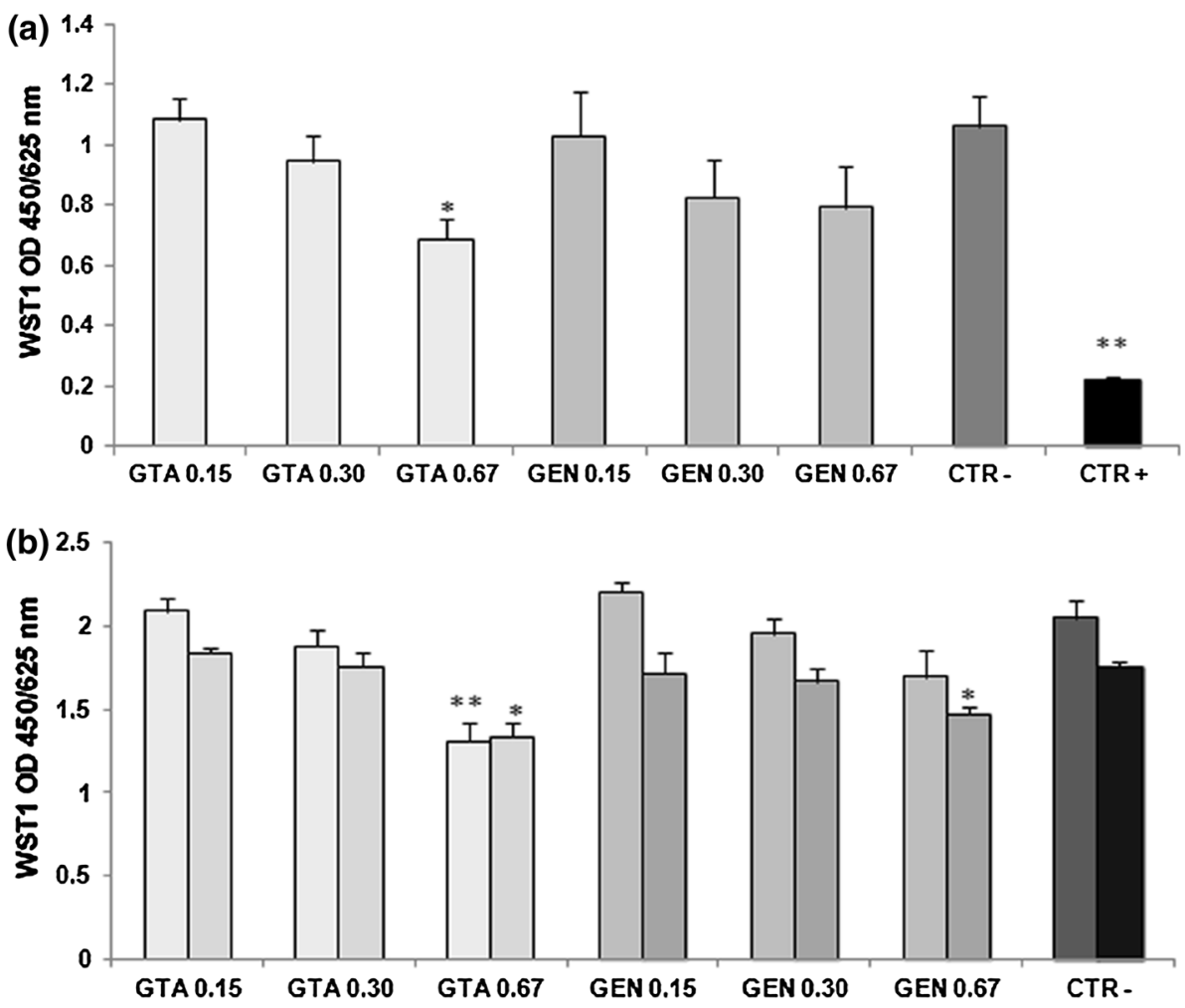

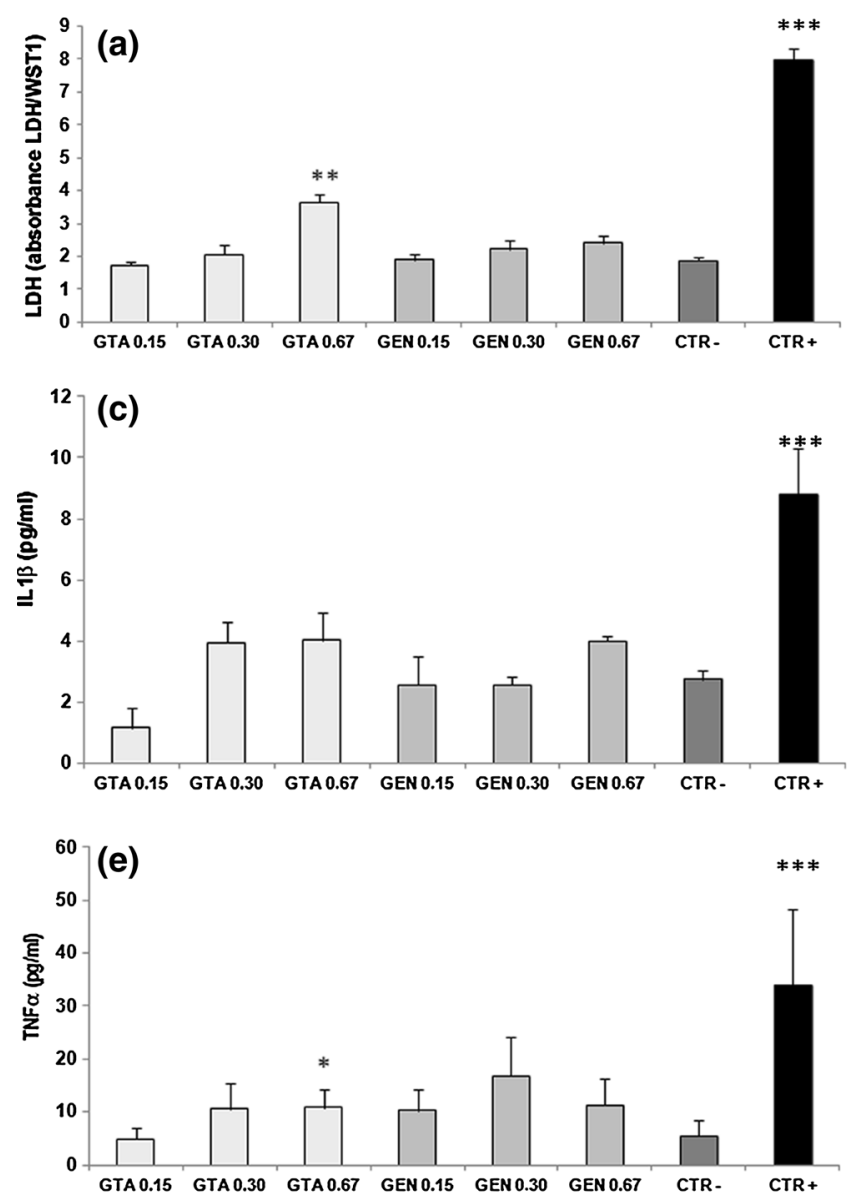

Fig. 7 Analysis of LDH (a-b), IL1 $\beta$ (c-d) and TNF $\alpha$ (e-f) production by BJ5ta culture on material samples and control after 72 h, 14 and 28 days. Results are the mean \pm SD (post hoc Dunnett

thermal stability of the films, in agreement with the values of $T_{\mathrm{D}}$ and $\Delta H_{\mathrm{D}}$ of the samples submitted to $\gamma$-irradiation which are close to those recorded on non-sterilized samples. FTIR analysis carried out on gelatin films before and after sterilization confirms that $\gamma$-irradiation does not affect the molecular structure of the protein. Both spectra reported in Fig. 5 show a broad band in the range of $3,600-2,700 \mathrm{~cm}^{-1}$, characteristic of amide A, and further bands in the range $1,700-1,000 \mathrm{~cm}^{-1}$. In particular, amide $\mathrm{I}$ at $1,647 \mathrm{~cm}^{-1}$, corresponding to stretching vibrations of peptide $\mathrm{C}=\mathrm{O}$ groups, amide II at $1,552 \mathrm{~cm}^{-1}$, deriving from $\mathrm{N}-\mathrm{H}$ bending and $\mathrm{C}-\mathrm{N}$ stretching, and amide III at $1,235 \mathrm{~cm}^{-1}$, assigned to $\mathrm{C}-\mathrm{O}$ stretching and $\mathrm{N}-\mathrm{H}$ bending vibrations [23]. Neither the position of the amide I band, which has been related to the triple helix content [24] nor the ratio of the intensities of amide III to $1,450 \mathrm{~cm}^{-1}$, which is considered to be a measure of preservation of integrity of triple helix [23], vary after sterilization in agreement with preservation of molecular structure.

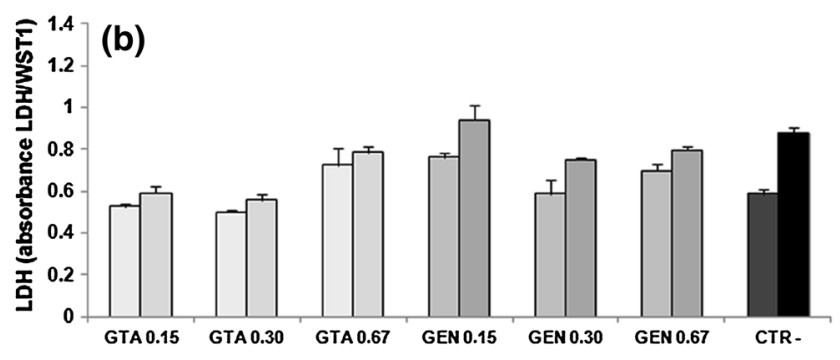

(d)
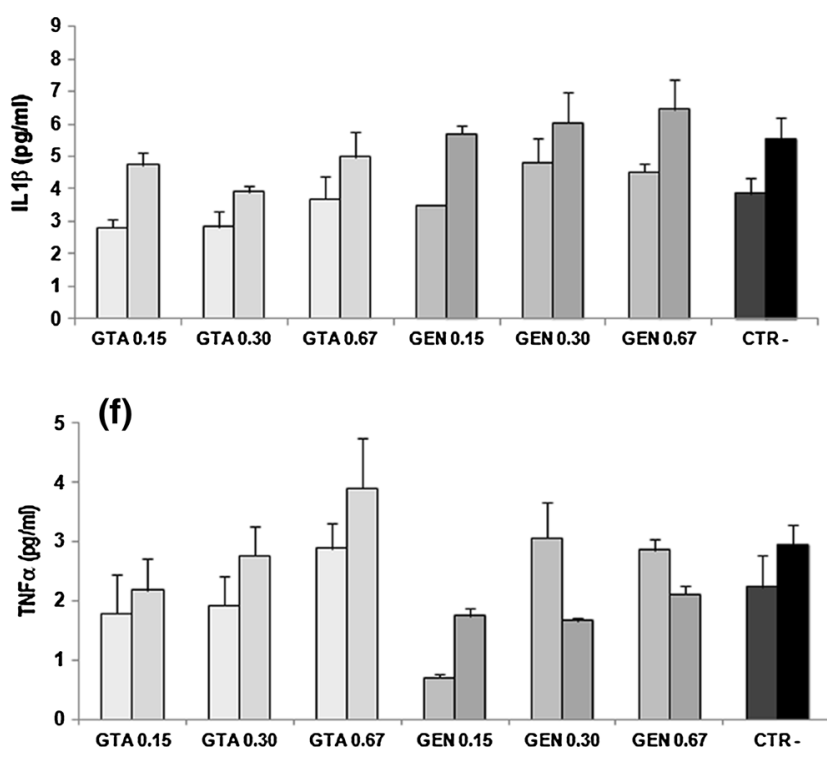

test: $* P<0.05 ; * * P<0.005 ; * * * P<0.0005)$. (a) $* * \mathrm{GTA} 0.67$ versus CTR $-; * * * \mathrm{CTR}+$ versus all samples. $(\mathbf{b}) * * * \mathrm{CTR}+$ versus all samples. (c) *GTA0.67 versus CTR-; ***CTR+ versus all samples

\subsection{In vitro study}

BJ-5ta cell line cultured on gelatin films crosslinked with GTA or GEN and controls was evaluated after 72 h, 14 and 28 days of culture. After $72 \mathrm{~h}$ of culture (Fig. 6a), the percentage of viability of cells grown on GTA0.15, GTA0.30, GEN0.15, GEN0.30 and GEN0.67 was higher than $70 \%$ (102, 89, 98, 81, and $75 \%$ respectively) compared to CTR-, showing no cytotoxic effects. At variance, GTA0.67 group showed significantly lower percentage of proliferation $(65 \%, P<0.05)$. CTR + group, as expected, showed a very significantly low percentage of proliferation when compared to all groups $(P<0.0005)$. At 14 and 28 days (Fig. $6 \mathrm{~b}$ ) GTA0.67 proliferation remained significantly lower, $P<0.005$, and $P<0.05$ respectively. At 28 days GEN0.67 proliferation was also lower than CTR $-(P<0.05)$.

The results of LDH, IL1 $\beta$ and TNF $\alpha$ showed that the levels of these parameters were related and inversely proportional (correlation Person test $P<0.0001$ ) to cell viability. LDH released by cells as a consequence of 

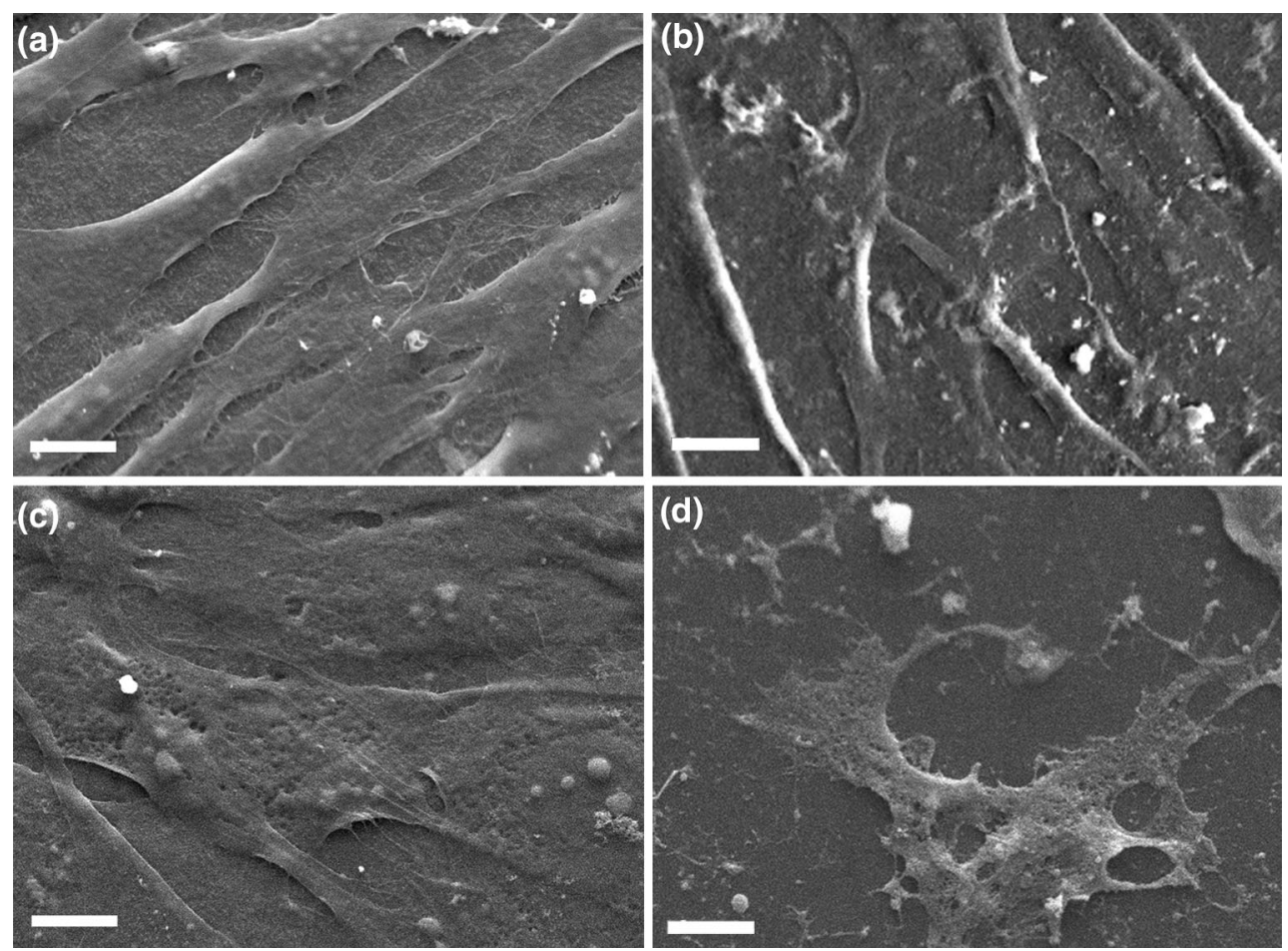

Fig. 8 SEM micrographs of fibroblast grown on (a) GEN0.15, (b) GTA0.15, (c) GEN0.67, (d) GTA0.67. Bars $=10 \mu \mathrm{m}$

cytoplasmatic membrane damage was measured in the culture medium. At 72 h GTA0.67 group showed significantly higher values of LDH in comparison to CTR- (Fig. 7a). At 14 and 28 days no differences were found among all groups (Fig. 7b). To assess if crosslinking agents had effects on the production of pro-inflammatory factors, IL $1 \beta$ and TNF $\alpha$ were measured in supernatants of experimental samples and controls. CTR + tested at $72 \mathrm{~h}$ showed significantly higher values of both IL1 $\beta$ and TNF $\alpha$ when compared to CTR$(P<0.0005)$. Regarding IL1 $\beta$ (Fig. 7c, d), no differences were found among groups and CTR-. At $72 \mathrm{~h} \mathrm{level} \mathrm{of} \mathrm{TNF} \alpha$ (Fig. 7e) in GTA0.67 group was significantly higher, when compared to CTR-, however at 14 and 28 days (Fig. 7f) TNF $\alpha$ was not different from CTR-.

At the end of the experimental time, the cells appeared well spread and attached on the films, as shown in Fig. 8a-d. The fibroblasts, rich of philopodia, are very numerous on the surface of all the samples, with the exception of GTA0.67 that displays a reduced amount of cells (Fig. 8d).

Results of WST1 test at the end of experimental times demonstrated a correlation between proliferation grade and crosslinker concentration. Genipin is considered a low-toxic crosslinking agent [25-27]. In the present work no significant negative effects on cell viability were found for GEN at the used concentration. On the contrary, GTA at the higher concentration showed negative effects even after extensive rinsing. The decrease in cell attachment and proliferation onto GTA0.67 does not seem to be associated to the residual GTA, but it may be related to the blocking of cell biding sites altered by the cross-linking process [28]. A possible explanation may also involve GTA alteration of integrin activity that regulates cell adhesion [29].

\section{Conclusions}

Although $\gamma$-irradiation causes a worsening of the mechanical properties of uncrosslinked gelatin films, it does not alter the mechanical response, thermal stability, as well as swelling and gelatin release of crosslinked films. It follows that $\gamma$ irradiation can be considered a useful method to sterilize gelatin based biomaterials without adverse effects. Moreover, the comparison of the data obtained on crosslinked films puts into evidence the greater values of elastic modulus of GTA films with respect to GEN films. In addition to the effects described above on viability in cell adhesion, GTA0.67 expresses a direct cytotoxic effect on the cells as demonstrated by elevated LDH and TNF at $72 \mathrm{~h}$, even if this effect is transient and disappears over time. However, the values of LDH and TNF $\alpha$ recorded for GTA0.67 indicate an acute and dose dependent cytotoxic effect of this crosslinking agent, and confirm the better biological performance of genipin treated materials.

Acknowledgments This work was financially supported by MIUR (FIRB Nº. RBAP10MLK7). 


\section{References}

1. Mano JF, Silva GA, Azevedo HS, Malafaya PB, Sousa RA, Silva SS, Boesel LF, Oliveira JM, Santos TC, Marques AP, Neves NM, Reis RL. Natural origin biodegradable systems in tissue engineering and regenerative medicine: present status and some moving trends. J R Soc Interface. 2007;4:999-1030.

2. Gómez-Guillén MC, Giménez B, López-Caballero ME, Montero MP. Functional and bioactive properties of collagen and gelatin from alternative sources: a review. Food Hydrocoll. 2011;25: 1813-27.

3. Veis A. The macromolecular chemistry of gelatin. New York: Academic; 1964.

4. Pezron I, Djabourov M, Leblond J. Conformation of gelatin chains in aqueous solutions: 1 . A light and small angle neutron scattering study. Polymer. 1991;32:3201-10.

5. Ross-Murphy SB. Structure and rheology of gelatin gels: recent progress. Polymer. 1992;33:2622-7.

6. Ackerman MS, Bhate M, Shenoy N, Beck K, Ramshaw JA, Brodsky B. Sequence dependence of the folding of collagen-like peptides single amino acids affect the rate of triple-helix nucleation. J Biol Chem. 1999;274:7668-73.

7. Bigi A, Panzavolta S, Rubini K. Relationship between triple helix content and mechanical properties of gelatin films. Biomaterials. 2004;25:5675-80.

8. Gornall JL, Terentjev EM. Helix-coil transition of gelatin: helical morphology and stability. Soft Matter. 2008;4:544-9.

9. Boanini E, Rubini K, Panzavolta S, Bigi A. Chemico-physical characterization of gelatin films modified with oxidized alginate. Acta Bio. 2010;6:383-8.

10. Hashim DM, Man YBC, Norakasha R, Shuhaimi M, Salmah Y, Syahariza ZA. Potential use of Fourier transform infrared spectroscopy for differentiation of bovine and porcine gelatins. Food Chem. 2010;118:856-60.

11. Noah EM, Chen J, Jiao X, Heschel I, Pallua N. Impact of sterilization on the porous design and cell behavior in collagen sponges prepared for tissue engineering. Biomaterials. 2002;23: 2855-61.

12. Wiegand C, Abel M, Ruth P, Wilhelms T, Schulze D, Norgauer J, Hipler UC. Effect of the sterilization method on the performance of collagen type I on chronic wound parameters in vitro. J Biomed Mater Res B: Appl Biomater. 2009;90B:710-9.

13. Burton B, Gaspar A, Josey D, Tupy J, Grynpas MD, Willett TL. Bone embrittlement and collagen modifications due to high-dose gamma-irradiation sterilization. Bone. 2014;61:71-81.

14. Panzavolta S, Torricelli P, Amadori S, Parrilli A, Rubini K, Bella ED, Fini M, Bigi A. 3D interconnected porous biomimetic scaffolds: in vitro cell response. J Biom Mat Res A. 2013;101A: 3560-70.

15. Gioffrè $\mathrm{M}$, Torricelli $\mathrm{P}$, Panzavolta $\mathrm{S}$, Rubini K, Bigi A. Role of $\mathrm{pH}$ on stability and mechanical properties of gelatin films. J Bioact Compat Polymers. 2012;27:57-67.
16. Dong GC, Chen HM, Yao CH. A novel bone substitute composite composed of tricalcium phosphate, gelatin and drynaria fortunei herbal extract. J Biomed Mater Res. 2008;84A:167-77.

17. Bigi A, Bracci B, Cojazzi G, Panzavolta S, Roveri N. Drawn gelatin films with improved mechanical properties. Biomaterials. 1998;19:2335-40.

18. Bigi A, Cojazzi G, Panzavolta S, Roveri N, Rubini K. Stabilization of gelatin films by crosslinking with genipin. Biomaterials. 2002;23:4827-32.

19. Bigi A, Cojazzi G, Panzavolta S, Roveri N, Rubini K. Mechanical and thermal properties of gelatin films at different degrees of glutaraldehyde crosslinking. Biomaterials. 2001;22:763-8.

20. Ofner CMIII, Bubnis WA. Chemical and swelling evaluations of amino group crosslinking in gelatin and modified gelatin matrices. Pharm Res. 1996;13:1821-7.

21. Hataka T, Sato H, Watanabe Y, Matsumoto M. Effect of formaldehyde on the physiochemical properties of soft gelatin capsule shells. Chem Pharm Bull. 1994;42:1138-42.

22. Tonda-Turo C, Gentile P, Saracino S, Chiono V, Nandagiri VK, Muzio G, Canuto RA, Ciardelli G. Comparative analysis of gelatin scaffolds crosslinked by genipin and silane coupling agent. Int J Biol Macromol. 2011;49:700-6.

23. Tronci G, Doyle A, Russell SJ, Wood DJ. Triple-helical collagen hydrogels via covalent aromatic functionalisation with 1,3phenylenediacetic acid. J Mater Chem B. 2013;1:5478-88.

24. Zhang L, Aksan A. Fourier transform infrared analysis of the thermal modification of human cornea tissue during conductive keratoplasty. Appl Spectrosc. 2010;64:23-9.

25. Fessel G, Cadby J, Wunderli S, van Weeren R, Snedeker JG. Dose- and time-dependent effects of genipin crosslinking on cell viability and tissue mechanics - toward clinical application for tendon repair. Acta Biomater. 2014;10(5):1897-906.

26. Sundararaghavan HG, Monteiro GA, Lapin NA, Chabal YJ, Miksan JR, Shreiber DI. Genipin-induced changes in collagen gels: correlation of mechanical properties to fluorescence. J Biomed Mater Res A. 2008;87(2):308-20.

27. Chang WH, Chang Y, Lai PH, Sung HW. A genipin-crosslinked gelatin membrane as wound-dressing material: in vitro and in vivo studies. J Biomater Sci. 2003;14(5):481-95 (Polymer Edition).

28. Gough JE, Scotchford CA, Downes S. Cytotoxicity of glutaraldehyde crosslinked collagen/poly(vinyl alcohol) films is by the mechanism of apoptosis. J Biomed Mater Res. 2002;61(1): 121-30.

29. Chen DC, Lai YL, Lee SY, Hung SL, Chen HL. Osteoblastic response to collagen scaffolds varied in freezing temperature and glutaraldehyde crosslinking. J Biomed Mater Res A. 2007;80(2): 399-409. 